\title{
ESTUDIO COMPARATIVO DE FACTORES \\ ANTROPOMÉTRICOS Y DE CONDICIÓN FíSICA EN JUGADORES JÓVENES DE VOLEIBOL COLOMBIANO
}

\section{COMPARATIVE STUDY ON ANTROPOMÉTRIC FACTORS AND PHYSICAL CONDITION IN YOUNG COLOMBIAN VOLLEYBALL PLAYERS}

\author{
Yennys González ${ }^{1}$, Silvia Sedano ${ }^{2}$, Jairo Fernández ${ }^{3}$, Hernando Díaz ${ }^{4}$
}

\begin{abstract}
${ }^{1}$ Doctora en Ciencias de la Actividad Física y del Deporte, Universidad Santo Tomás, Grupo GICAEDS, Carrera 56 № $153-$ 15 Apto. 1102 Torre 2 Edificio Reserva de Colina, Bogotá D.C. Colombia, e-mail: yennysgonzalez@usantotomas.edu.co, yego61@yahoo.es; ${ }^{2}$ Doctora en Ciencias de la Actividad Física y del Deporte, Universidad Europea Miguel de Cervantes, Laboratorio de fisiología, Valladolid-España. C/Padre Julio Chevalier, 2. 47017 Valladolid, España, e-mail: ssedano@uemc. es; ${ }^{3}$ Magister Fisiología Universidad Claude Bernard, Lyon Francia, Universidad Pedagógica Nacional, Bogotá-Colombia, Docente Planta. Calle 72 № 11-86, e-mail: jairofdz@pedagogica.edu.co; ${ }^{4}$ Especialista en Administración Deportiva, Universidad Santo Tomás, Grupo GICAEDS, Carrera 98 № 147-61, Bogotá, D.C.-Colombia, e-mail: jesusdiaz@usantotomas.edu.co
\end{abstract}

Rev. U.D.C.A Act. \& Div. Cient. 17(1): 53-63, Enero-Junio, 2014

\section{RESUMEN}

El propósito principal de esta investigación fue realizar un análisis descriptivo para valorar la influencia que, sobre distintas variables de carácter antropométrico y de condición física, tienen factores como la categoría de edad, el nivel competitivo y la posición ocupada en el terreno de juego, en jugadores jóvenes de Voleibol. Por otra parte, determinar las correlaciones existentes entre las variables antropométricas, como también entre las capacidades motrices de fuerza explosiva y de agilidad. La muestra, se conformó por 267 jugadores hombres, pertenecientes a la liga de Cundinamarca $(n=131)$ y a colegios distritales de Bogotá $(n=136)$. Se registraron un total de 11 medidas antropométricas (masa corporal, talla, masa grasa, envergadura y siete pliegues) y tres pruebas de campo. En general, se observó una influencia notable de la edad cronológica en la evolución de las variables evaluadas; dicha influencia se consideró superior a la ejercida por el nivel competitivo, siendo evidente en el caso de los jugadores de menor nivel, posiblemente, por factores del nivel socio-económico de los jugadores, aunque estadísticamente no existan diferencias significativas. Respecto al porcentaje de masa grasa, ni el nivel competitivo ni la edad manifiestan tener influencia en la evolución de esta variable. Además, se observa un perfil antropométrico específico en los centrales, perfil vinculado a las exigencias específicas de esta posición. En relación a las pruebas de condición física, los colocadores obtuvieron los mejores resultados, teniendo relación con las exigencias tácticas de este deporte.
Palabras clave: Voleibol, antropometría, composición corporal, fuerza explosiva y agilidad.

\section{SUMMARY}

The main purpose of this research was to carry out a descriptive analysis to assess the influence on anthropometric characters variables and physical condition that factors such as age, competitive level and position performed on the court, have in young volleyball players. Also to determine the correlation between both, anthropometric variables and motor skills agility and explosive power. The sample consisted of 267 male players belonging to the Cundinamarca League $(n=131)$ and Bogotá district schools $(n=136)$. A total of 11 anthropometric measures were performed (body mass, height, fat mass, size and seven-fold) and three field tests. Mostly, there is a large influence of chronological age in the evolution of the variables. This influence show to be superior to that exerted by the competitive level, being evident in the case of lower level players, due to player's socio- economic level factors, even though there is not statistically significant differences. Regarding the percentage of fat mass, neither, the competitive level not the age, seemed to influence the evolution of this variable. Moreover, it was detected a specific anthropometric profile in central player, profile linked to the specific requirements of this position. Relative to physical fitness tests, setters performed best, this may be due to sport tactics requirements. 
Key words: Volleyball, anthropometry, body composition, explosive strength and agility.

\section{INTRODUCCIÓN}

El rendimiento en el voleibol está basado en una compleja e intrincada diversidad de variables, que incluye factores psicológicos, fisiológicos, físicos y morfológicos (Gaurav et al. 2011) y la literatura ha resaltado la importancia de los factores que son considerados relevantes en el rendimiento en el voleibol (Peña et al. 2013). En este deporte, considerado intermitente o intervalado, se requiere que los jugadores completen ejercicios de alta intensidad, en acciones cortas frecuentes, seguidos por acciones de baja intensidad (Torres et al. 1993; Gabbett, 2008). Las acciones en las que el ejercicio es de alta intensidad, relacionadas con la duración total del juego, aproximadamente 90 minutos, requiere que los jugadores tengan bien desarrollados los sistemas de obtención de energía, tanto aeróbico como anaeróbico. En consecuencia, los jugadores de voleibol requieren velocidad, agilidad, potencia en tren superior e inferior y $\mathrm{VO}_{2 \max }$ bien desarrollados (Gabbett, 2008). Varios estudios han documentado las características fisiológicas, de condición física y antropométrica de los jugadores de voleibol. Smith et al. (1992) hallaron una diferencia significativa en la altura de los saltos de bloqueo y remate, en la velocidad en 20 metros, en el $\mathrm{VO}_{2 \max }$ a favor de los jugadores de categoría nacional, sugiriendo que las capacidades fisiológicas juegan un papel importante en la preparación y en la selección de competidores de élite. Por otro lado, un estudio reciente de Cabral et al. (2013), específicamente sobre la variable antropométrica talla, la cual, se considera de gran importancia en el rendimiento de este deporte, indica que los jugadores jóvenes brasileños presentan una media similar a la de los de categoría adulta.

Análisis sobre características antropométricas y de condición física son indispensables, inicialmente, para orientar el proceso de selección de nuevos jugadores (Toledo et al. 2000); posteriormente, esta información será utilizada para especializar por posiciones de juego y en el control del entrenamiento deportivo (Durkovic et al. 2012). Haciendo una revisión en las principales bases de datos (Sportdiscus, Proquest, Scielo), no se encontraron estudios sobre esta temática realizados en Colombia.

\section{MATERIALES Y MÉTODOS}

Muestra: La muestra, se conformó por 267 sujetos, distribuidos en dos niveles de competencia y en tres categorías según la edad:

Grupo de voleibolistas 1 (GV1) ( $\mathrm{N}=131)$ : Varones voleibolistas con licencia federativa en vigor departamental, inscri- tos a la Liga de Cundinamarca (Colombia), en la temporada 2010-2011, pertenecientes a tres categorías: Infantil (13 y 14 años), Pre-Juvenil (15 y 16 años) y Juvenil (17 y 18 años). Dichos jugadores entrenaban una media de seis horas a la semana y participaban en tres concentraciones anuales en fines de semana y cuatro torneos abiertos de categoría única. En este grupo, se evaluaron 57 Rematadores (R4), 36 Centrales o R3 y 38 Colocadores (C2).

Grupo de jugadores de voleibol 2 (GV2) ( $\mathrm{N}=136)$ : Varones con licencia federativa en vigor inter-colegiada distrital (Bogotá-Colombia), en la temporada 2010-2011, pertenecientes a tres categorías: Infantil (13 y 14 años), Pre-Juvenil (15 y 16 años) y Juvenil (17 y 18 años). Dichos jugadores de voleibol entrenaban una media de cuatro horas a la semana, con uno o dos partidos por semana. En este grupo, se evaluaron 66 Rematadores (R4), 39 Centrales o R3 y 31 Colocadores (C2).

Material: Plicómetro especial tipo Harpenden modelo HSK$\mathrm{BI}^{\circledR}$, que ejerce una presión normalizada sobre los pliegues de $10 \mathrm{~g} / \mathrm{mm}^{2}$, con precisión $0,20 \mathrm{~mm}$. Intervalo de medición de 0 a $80 \mathrm{~mm}$. Calibre de campo marca L\&W tools ${ }^{\circledR}$, con una precisión de $1 \mathrm{~mm}$. Intervalo de medición de 0,5 a $110 \mathrm{~cm}$. Báscula TANITA BF- $666^{\circledR}$, con una precisión de $0,1 \mathrm{~kg}$. Intervalo de medición de 0 a $150 \mathrm{~kg}$. Tallímetro Holtain ${ }^{\circledR}$, con una precisión de $0,1 \mathrm{~cm}$. Intervalo de medición de 60 a $209 \mathrm{~cm}$. Una escala métrica fijada en la pared de $2 \mathrm{~m}$ de altura por $2,5 \mathrm{~m}$ de ancho, con marcas de un centímetro horizontales y verticales. Sistema de fotocélulas de luz infrarroja tipo reflejo, para la medición de tiempos con precisión de $100 \mathrm{~Hz}$, modelo WL34-R240. Marca Sick ${ }^{\circledR}$ Potencia de encendido: Relevo, SPDT. Aislado. Max. Frecuencia de encendido: 10/s. Tiempo de demora: tabla, ajustable 0,5 a 10 s. Optojump Microgate ${ }^{\circledR}$ conectado a un ordenador portatil Toshiba Satellite, con el sistema operativo Windows 7 y el software Optojump ${ }^{\circledR}$, diseñado específicamente para el registro de los datos procedentes del Optojump. Precisión de 1/1000 segundos.

Para el registro y tratamiento de datos, se utilizó Ordenador Toshiba Satellite, con el sistema operativo Windows 7. Una hoja de cálculo Excel para Windows 7 y el programa estadístico SPSS. Versión 19.

Procedimiento: Todas las mediciones, se realizaron durante la temporada competitiva de todos los clubes y colegios, en el primer trimestre del 2011. Después de entregar la información a los entrenadores de los diferentes clubes y colegios de voleibol, se tramitaron los permisos con los padres de familia, ya que los participantes eran menores de edad. Se citaron a todos los deportistas en la Facultad de Educación Física de la Universidad Pedagógica Nacional a una reunión, donde se les explicó directamente, los objetivos, la metodología y riesgos de la investigación. Además, se les informó 
del procedimiento específico de la parte experimental y se les dio la oportunidad de realizar preguntas.

La totalidad de las pruebas antropométricas, de campo y de laboratorio, se desarrollaron en una sola sesión de evaluación en ese orden, a la misma hora por la mañana y bajo los mismos parámetros. Un evaluador experimentado realizó las mediciones antropométricas necesarias para la determinación de la composición corporal, con la ayuda de una asistente. Siguiendo los protocolos de medidas antropométricas establecidos por la International Society for the Advancement of Kinanthropometry (ISAK) Mazza (2010), tras efectuar la adecuada calibración de los instrumentos, se tomaron las siguientes medidas: Talla, masa corporal, altura trocantérea y siete pliegues - tríceps, subescapular, bíceps, suprailíaco, abdominal, muslo anterior, medial de la pierna-. El porcentaje de grasa, se calculó a través de la ecuación propuesta por Faulkner (1968). En cuanto a las pruebas de campo y de laboratorio, todos los grupos realizaron un calentamiento estandarizado de 20 minutos. Las pruebas de campo fueron la prueba de fuerza explosiva del tren superior - Test Lanzamiento balón medicinal- y de agilidad -T-Test-, en ese orden y, finalmente, las pruebas de fuerza explosiva en el laboratorio, salto con contra movimiento -CMJ- y salto Abalakov -ABK-.

Prueba T-Test (Pauole et al. 2000): Esta prueba, se realizó ubicando cuatro conos separados $5 \mathrm{~m}$ entre sí, formando la letra "T". El jugador de voleibol decidía cuando iniciar su movimiento; partía del cono de la "base de la T" en línea recta a tocar con la mano el primer cono; luego, seguía a tocar el cono de la izquierda regresaba al cono central, seguía al cono de la derecha y, finalmente, terminaba en el cono del inicio. Se realizaron dos intentos variando la dirección de izquierda o derecha del primer cono, de forma aleatoria con el sistema de dos subgrupos. Se tomó el mejor registro como válido para el análisis estadístico. El intervalo de recuperación entre intentos fue de tres minutos. Todos los tiempos fueron cronometrados con el sistema de fotocélulas de sistema infrarrojo, ubicadas en el primer cono ("base de la T").

Variante: Lanzamiento de balón medicinal con apoyo de dos rodillas en el suelo (LBM) (Martínez, 2012): Esta prueba, se efectuó ubicando una colchoneta en el piso, para que los jugadores de voleibol se arrodillaran en el borde de la misma. Estos jugadores debían tomar un balón medicinal de $3 \mathrm{~kg}$ por encima de la cabeza, sujetándolo con las dos manos y lanzándolo lo más lejos posible, usando únicamente la fuerza de los brazos. Para la realización del movimiento, se permitía flexionar los codos. Cada deportista contó con dos intentos, con un intervalo de recuperación entre ellos, de tres minutos. Un evaluador experimentado tomaba como referencia para la medición, el lugar de bote del balón. El mejor registro fue utilizado para el análisis estadístico.
Salto con contramovimiento (CMJ) (Bosco, 1994): Para la realización de esta prueba, el deportista se ubicó entre las dos barras del Optojump, partiendo de una extensión de rodillas en bipedestación, con las manos sujetas en las caderas, donde habían de permanecer desde la posición inicial hasta la final. Posteriormente, realizaba un movimiento rápido de flexo-extensión de las rodillas, previo al salto vertical máximo. Se realizaron dos intentos, con un intervalo de tres minutos entre los dos saltos, seleccionando el mejor registro para el análisis estadístico.

Salto Abalakov (ABK) (Bosco, 1994): Al igual que en CMJ, para iniciar la prueba el deportista, se ubicó entre las dos barras del Optojump, partiendo de una extensión de rodillas en bipedestación, pero con las manos libres a los lados del cuerpo. Debía realizar un movimiento rápido de flexo-extensión de las rodillas, utilizando el movimiento de los brazos de abajo hacia arriba, como impulso, para ejecutar un salto vertical máximo. Se realizaron dos intentos separados por tres minutos de recuperación, seleccionando el mejor registro para el análisis estadístico. Entre el CMJ y el ABK el intervalo de recuperación fue de tres minutos, en los que se realizaban estiramientos activos.

Análisis Estadístico: Para determinar la normalidad de la muestra, se utilizó la prueba no paramétrica de KolmogorovSmirnov, para una muestra. Se calcularon los estadísticos descriptivos de las diferentes variables analizadas para cada grupo en función del nivel, la categoría y la posición habitual de juego. A la hora de comparar las diferentes variables en función de los distintos factores, se empleó un análisis de varianza de un sólo factor-ANOVA-, con pruebas post-hoc de Bonferroni. Para ANOVA, el nivel, la categoría y la posición habitual de juego, se utilizaron como variables independientes, mientras que la masa corporal, la talla, la altura trocantérea, el porcentaje de masa grasa, los tiempos en las pruebas de velocidad y agilidad y la altura en CMJ y ABK, se emplearon como variables dependientes. Para determinar las correlaciones existentes entre variables, se calcularon las correlaciones bivariadas, a través del coeficiente de correlación de Pearson. Para todos los análisis las diferencias son significativas cuando $p<0.05$.

\section{RESULTADOS Y DISCUSIÓN}

Resultados obtenidos en función de las categorías: En la tabla 1, se muestran los resultados obtenidos en el análisis comparativo de variables entre las categorías infantil, pre-juvenil y juvenil. En este sentido, ANOVA reflejó la existencia de diferencias significativas entre categorías, para las variables de masa corporal, talla, envergadura, LBM, CMJ y ABK. Dentro de las variables antropométricas, el análisis post-hoc de Bonferroni localizó dichas diferencias entre infantiles y juveniles, para la masa corporal y entre infantiles y pre-juveniles 
e infantiles y juveniles, para la talla y la envergadura. Por otro lado, en CMJ y ABK existían diferencias significativas entre todas las categorías al igual que LBM, siendo en ambos casos, los juveniles los que obtenían los resultados más elevados y los infantiles los que obtenían los más bajos.
Resultados obtenidos en función de las categorías dentro de cada nivel de competencia: Dentro de GV1, ANOVA reflejó la existencia de diferencias significativas en las variables de masa corporal, talla, envergadura, T-Test, LBM, CMJ y ABK. Dentro de las variables antropométricas, las pruebas

Tabla 1. Resultados y análisis comparativo de todas las variables en función de las categorías.

\begin{tabular}{|c|c|c|c|c|}
\hline VARIABLE & CATEGORÍA & $\mathrm{N}$ & MEDIA \pm SD & F - RATIO \\
\hline \multirow{3}{*}{ Masa corporal (kg) } & Infantil & 108 & $53,74 \pm 33,48 a$ & \multirow{3}{*}{$5,94 *$} \\
\hline & Pre-juvenil & 111 & $60,94 \pm 9,73_{\mathrm{ab}}$ & \\
\hline & Juvenil & 48 & $66,52 \pm 10,90_{b}$ & \\
\hline \multirow{3}{*}{ Talla (cm) } & Infantil & 108 & $160,49 \pm 9,79 a$ & \multirow{3}{*}{$68,96 *$} \\
\hline & Pre-juvenil & 111 & $171,33 \pm 6,89 \mathrm{~b}$ & \\
\hline & Juvenil & 48 & $174,19 \pm 6,37 \mathrm{~b}$ & \\
\hline \multirow{3}{*}{ Envergadura (cm) } & Infantil & 108 & $161,89 \pm 11,25 a$ & \multirow{3}{*}{$60,32 *$} \\
\hline & Pre-juvenil & 111 & $173,53 \pm 7,83 b$ & \\
\hline & Juvenil & 48 & $176,58 \pm 7,40 \mathrm{~b}$ & \\
\hline \multirow{3}{*}{ Masa Grasa (\%) } & Infantil & 108 & $14,08 \pm 8,15$ & \multirow{3}{*}{0,03} \\
\hline & Pre-juvenil & 111 & $13,87 \pm 6,90$ & \\
\hline & Juvenil & 48 & $14,18 \pm 71,94$ & \\
\hline \multirow{3}{*}{ T-Test (s) } & Infantil & 108 & $8,53 \pm 2,37$ & \multirow{3}{*}{2,05} \\
\hline & Pre-juvenil & 111 & $8,62 \pm 1,16$ & \\
\hline & Juvenil & 48 & $7,98 \pm 1,99$ & \\
\hline \multirow{3}{*}{$\operatorname{LBM}(\mathrm{m})$} & Infantil & 108 & $3,80 \pm 0,81_{a}$ & \multirow{3}{*}{$82,72 *$} \\
\hline & Pre-juvenil & 111 & $4,79 \pm 0,84 b$ & \\
\hline & Juvenil & 48 & $5,49 \pm 0,71_{c}$ & \\
\hline \multirow{3}{*}{ CMJ $(\mathrm{cm})$} & Infantil & 108 & $28,57 \pm 6,72 \mathrm{a}$ & \multirow{3}{*}{$38,10 *$} \\
\hline & Pre-juvenil & 111 & $34,10 \pm 5,80 b$ & \\
\hline & Juvenil & 48 & $36,89 \pm 5,45 c$ & \\
\hline \multirow{3}{*}{$\mathrm{ABK}(\mathrm{cm})$} & Infantil & 108 & $32,67 \pm 7,40 a$ & \multirow{3}{*}{$48,82 *$} \\
\hline & Pre-juvenil & 111 & $39,75 \pm 6,60 \mathrm{~b}$ & \\
\hline & Juvenil & 48 & $42,97 \pm 5,73 c$ & \\
\hline
\end{tabular}

*Diferencias significativas: $p<0.05$. Las medias que en la misma columna, para la misma variable tienen el mismo subíndice no son estadísticamente diferentes $(p<0.05)$.

post-hoc localizaron las diferencias entre todas las categorías en el caso de la masa corporal, siendo los juveniles los que presentaban una masa mayor y los infantiles, menor y entre los infantiles y los pre-juveniles y juveniles, tanto para la talla como para la envergadura. En el caso de las pruebas de campo, las diferencias se localizan entre los infantiles y los pre- juveniles y juveniles, tanto para el T-Test como para LBM, CMJ y ABK. En los tres casos son los infantiles los que obtienen más bajos resultados. Dentro de GV2, ANOVA mostró la existencia de diferencias significativas en las variables de talla, envergadura, LBM, CMJ y ABK. Por un lado, las diferencias se localizaron entre los infantiles y los pre- 
juveniles y juveniles, tanto en talla como en envergadura. Por el otro, en el caso del LBM Y CMJ aparecían diferencias entre todos los grupos, siendo los juveniles los que obtenían los valores más elevados; sin embargo, en ABK, las diferencias aparecían entre los infantiles y los juveniles y entre éstos y los pre-juveniles, siendo los juveniles los que de nuevo obtenían los valores más elevados.

Resultados obtenidos en función del nivel competitivo: ANOVA no mostró la existencia de diferencias significativas; no obstante, los resultados del GV2 fueron más elevados en las variables de masa corporal, talla, envergadura, T-Test, LBM, CMJ y ABK. Por su parte, GV1 obtuvo peores registros en la variable de masa grasa (\%).

Resultados obtenidos en función del nivel competitivo dentro de cada categoría: En la tabla 2, ANOVA solamente mostró la existencia de diferencias significativas en la variable ABK, para la categoría de Infantiles, siendo más elevados los resultados de GV2. Cabe anotar que en las otras categorías, pre-juvenil y juvenil, ANOVA no evidenció diferencias significativas, en ninguna de las variables analizadas.

Tabla 2. Resultados y análisis comparativo de todas las variables en función del nivel competitivo dentro de la categoría de Infantiles.

\begin{tabular}{|c|c|c|c|c|}
\hline \multicolumn{5}{|c|}{ INFANTILES } \\
\hline VARIABLE & NIVEL & $\mathrm{N}$ & MEDIA \pm SD & F-RATIO \\
\hline \multirow{2}{*}{ Masa corporal (kg) } & GV1 & 64 & $49,40 \pm 9,76$ & \multirow{2}{*}{2,681} \\
\hline & GV2 & 44 & $60,05 \pm 50,80$ & \\
\hline \multirow{2}{*}{ Talla $(\mathrm{cm})$} & GV1 & 64 & $159,83 \pm 9,89$ & \multirow{2}{*}{0,715} \\
\hline & GV2 & 44 & $161,45 \pm 9,67$ & \\
\hline \multirow{2}{*}{ Envergadura (cm) } & GV1 & 64 & $161,35 \pm 11,56$ & \multirow{2}{*}{0,345} \\
\hline & GV2 & 44 & $162,65 \pm 10,89$ & \\
\hline \multirow{2}{*}{ Masa grasa (\%) } & GV1 & 64 & $14,54 \pm 7,87$ & \multirow{2}{*}{0,494} \\
\hline & GV2 & 44 & $13,41 \pm 8,59$ & \\
\hline \multirow{2}{*}{ T-Test (s) } & GV1 & 64 & $8,33 \pm 2,64$ & \multirow{2}{*}{1,131} \\
\hline & GV2 & 44 & $8,83 \pm 1,89$ & \\
\hline \multirow{2}{*}{$\operatorname{LBM}(\mathrm{m})$} & GV1 & 64 & $3,78 \pm 0,83$ & \multirow{2}{*}{2,145} \\
\hline & GV2 & 44 & $3,82 \pm 0,80$ & \\
\hline \multirow{2}{*}{$\mathrm{CMJ}(\mathrm{cm})$} & GV1 & 64 & $26,82 \pm 5,75$ & \multirow{2}{*}{$11,748 *$} \\
\hline & GV2 & 44 & $31,12 \pm 7,24$ & \\
\hline \multirow{2}{*}{$\mathrm{ABK}(\mathrm{cm})$} & GV1 & 64 & $31,62 \pm 8,55$ & \multirow[b]{2}{*}{3,236} \\
\hline & GV2 & 44 & $34,20 \pm 8,32$ & \\
\hline
\end{tabular}

*Diferencias significativas: $\mathrm{p}<0.05$.

Resultados obtenidos en función de la posición habitual de juego: En la tabla 3, se reflejan los resultados del análisis comparativo de las variables en función de las posiciones habituales de juego. ANOVA revela la existencia de diferencias significativas en las variables antropométricas. En el caso de la masa corporal, las diferencias se localizan entre la posición R4 y la R3 y entre R3 y C2. Por su parte, en la talla y en la envergadura, las diferencias significativas aparecen entre R4 y R3.
Resultados obtenidos en función del nivel de competencia dentro de cada posición de juego: Dentro de la posición R4, se observan diferencias significativas únicamente en las variables CMJ, siendo mejor el resultado en GV2. Con respecto a las otras posiciones de juego, $\mathrm{R} 3$ y C2 no se observaron diferencias significativas, entre niveles de competencia. 
Tabla 3. Resultados y análisis comparativo de todas las variables en función de las posiciones habituales de juego.

\begin{tabular}{|c|c|c|c|c|}
\hline VARIABLE & POSICIÓN & $\mathrm{N}$ & MEDIA \pm SD & F- RATIO \\
\hline \multirow{3}{*}{ Masa corporal (kg) } & $\mathrm{R} 4$ & 123 & $56,13 \pm 10,41 a$ & \multirow{3}{*}{$5,10 *$} \\
\hline & R3 & 75 & $66,15 \pm 39,47 \mathrm{~b}$ & \\
\hline & $\mathrm{C} 2$ & 69 & $56,47 \pm 11,02 a$ & \\
\hline \multirow{3}{*}{ Talla $(\mathrm{cm})$} & $\mathrm{R} 4$ & 123 & $165,74 \pm 8,89 a$ & \multirow{3}{*}{$5,60 *$} \\
\hline & R3 & 75 & $170,53 \pm 11,46 \mathrm{~b}$ & \\
\hline & $\mathrm{C} 2$ & 69 & $167,19 \pm 9,41_{a b}$ & \\
\hline \multirow{3}{*}{ Envergadura (cm) } & $\mathrm{R} 4$ & 123 & $167,30 \pm 10,02 \mathrm{a}$ & \multirow{3}{*}{$5,65 *$} \\
\hline & R3 & 75 & $172,73 \pm 13,01_{b}$ & \\
\hline & $\mathrm{C} 2$ & 69 & $169,39 \pm 10,27 \mathrm{ab}$ & \\
\hline \multirow{3}{*}{ Masa Grasa (\%) } & $\mathrm{R} 4$ & 123 & $13,80 \pm 7,65$ & \multirow{3}{*}{0,25} \\
\hline & R3 & 75 & $14,53 \pm 8,53$ & \\
\hline & $\mathrm{C} 2$ & 69 & $13,81 \pm 5,71$ & \\
\hline \multirow{3}{*}{ T-Test (s) } & $\mathrm{R} 4$ & 123 & $8,26 \pm 2,29$ & \multirow{3}{*}{1,67} \\
\hline & R3 & 75 & $8,77 \pm 1,48$ & \\
\hline & $\mathrm{C} 2$ & 69 & $8,52 \pm 1,41$ & \\
\hline \multirow{3}{*}{$\operatorname{LBM}(\mathrm{m})$} & $\mathrm{R} 4$ & 123 & $4,44 \pm 1,02$ & \multirow{3}{*}{1,44} \\
\hline & R3 & 75 & $4,48 \pm 1,08$ & \\
\hline & $\mathrm{C} 2$ & 69 & $4,70 \pm 0,99$ & \\
\hline \multirow{3}{*}{$\mathrm{CMJ}(\mathrm{cm})$} & R4 & 123 & $32,01 \pm 6,93$ & \multirow{3}{*}{0,58} \\
\hline & R3 & 75 & $32,24 \pm 7,58$ & \\
\hline & $\mathrm{C} 2$ & 69 & $33,13 \pm 6,21$ & \\
\hline \multirow{3}{*}{$\mathrm{ABK}(\mathrm{cm})$} & $\mathrm{R} 4$ & 123 & $37,00 \pm 8,07$ & \multirow{3}{*}{2,64} \\
\hline & R3 & 75 & $36,54 \pm 8,31$ & \\
\hline & $\mathrm{C} 2$ & 69 & $39,32 \pm 6,99$ & \\
\hline
\end{tabular}

*Diferencias significativas: $\mathrm{p}<0.05$. Las medias que en la misma columna, para la misma variable tienen el mismo subíndice no son estadísticamente diferentes $(\mathrm{p}<0.05)$.

Resultados obtenidos de las correlaciones de todas las variables en función del nivel de competencia: En la tabla 4, en el grupo GV1, se observa la existencia de una correlación positiva y estadísticamente significativa entre la masa corporal, por un lado y la talla, la envergadura y el porcentaje de grasa, por el otro; sin embargo, esta variable se correlaciona de manera negativa con el T-Test, al igual que ocurre con la talla o la envergadura. Por otro lado, tanto talla como envergadura o masa corporal, correlacionan, de manera sig- nificativa y positiva, con CMJ y ABK. Existe una correlación negativa y significativa entre la masa grasa y las pruebas $\mathrm{T}$ Test, ABK y CMJ. En el grupo GV2 existen correlaciones positivas y estadísticamente significativas entre la masa corporal y la masa grasa, entre la talla y la envergadura, entre la talla y la envergadura y CMJ y ABK y entre el T-Test y el LBM. Por otro lado, se observan correlaciones negativas entre el \% grasa y el T-Test, CMJ y ABK, aunque no llegan a alcanzar significación estadística. 
Tabla 4. Resultados obtenidos en el análisis de correlaciones entre variables en jugadores de voleibol de GV1.

\begin{tabular}{|c|c|c|c|c|c|c|c|c|}
\hline \multicolumn{9}{|c|}{ GV1 } \\
\hline VARIABLE & $\begin{array}{c}\text { MASA } \\
\text { CORPORAL }\end{array}$ & TALLA & $\begin{array}{l}\text { ENVERGA- } \\
\text { DURA }\end{array}$ & $\begin{array}{c}\text { MASA } \\
\text { GRASA } \\
(\%)\end{array}$ & T-TEST & LBM & CMJ & ABK \\
\hline Masa Corporal & $\begin{array}{l}1 \\
131\end{array}$ & $\begin{array}{l}, 719 * * \\
, 000 \\
131\end{array}$ & $\begin{array}{l}, 730 * * \\
, 000 \\
131\end{array}$ & $\begin{array}{l}, 270 * * \\
, 002 \\
131\end{array}$ & $\begin{array}{l}-, 254 * * \\
, 003 \\
131\end{array}$ & $\begin{array}{l}, 030 \\
, 730 \\
131\end{array}$ & $\begin{array}{l}, 367 * * \\
, 000 \\
131\end{array}$ & $\begin{array}{l}, 383 * * \\
, 000 \\
131\end{array}$ \\
\hline Talla & $\begin{array}{l}, 719 * * \\
, 000 \\
131\end{array}$ & $\begin{array}{l}1 \\
131\end{array}$ & $\begin{array}{l}, 947 * * \\
, 000 \\
131\end{array}$ & $\begin{array}{l}-, 007 \\
, 936 \\
131\end{array}$ & $\begin{array}{l}-, 418^{* *} \\
, 000 \\
131\end{array}$ & $\begin{array}{l}-, 031 \\
, 726 \\
131\end{array}$ & $\begin{array}{l}, 575 * * \\
, 000 \\
131\end{array}$ & $\begin{array}{l}, 594 * * \\
, 000 \\
131\end{array}$ \\
\hline Envergadura & $\begin{array}{l}, 730 * * \\
, 000 \\
131\end{array}$ & $\begin{array}{l}, 947 * * \\
, 000 \\
131\end{array}$ & $\begin{array}{l}1 \\
131\end{array}$ & $\begin{array}{l}-, 003 \\
, 975 \\
131\end{array}$ & $\begin{array}{l}-, 409 * * \\
, 000 \\
131\end{array}$ & $\begin{array}{l}-, 012 \\
, 894 \\
131\end{array}$ & $\begin{array}{l}, 548 * * \\
, 000 \\
131\end{array}$ & $\begin{array}{l}, 564 * * \\
, 000 \\
131\end{array}$ \\
\hline $\begin{array}{l}\text { Masa grasa } \\
(\%)\end{array}$ & $\begin{array}{l}, 270 * * \\
, 002 \\
131\end{array}$ & $\begin{array}{l}-, 007 \\
, 936 \\
131\end{array}$ & $\begin{array}{l}-, 003 \\
, 975 \\
131\end{array}$ & $\begin{array}{l}1 \\
131\end{array}$ & $\begin{array}{l}-, 274 * * \\
, 002 \\
131\end{array}$ & $\begin{array}{l}, 169 \\
, 054 \\
131\end{array}$ & $\begin{array}{l}-, 257 * * \\
, 004 \\
131\end{array}$ & $\begin{array}{l}-, 250 * * \\
, 003 \\
131\end{array}$ \\
\hline T-Test & $\begin{array}{l}-, 254 * * \\
, 003 \\
131\end{array}$ & $\begin{array}{l}-, 418^{* *} \\
, 000 \\
131\end{array}$ & $\begin{array}{l}-, 409 * * \\
, 000 \\
131\end{array}$ & $\begin{array}{l}, 274 * * \\
, 002 \\
131\end{array}$ & $\begin{array}{l}1 \\
131\end{array}$ & $\begin{array}{l}, 005 \\
, 952 \\
131\end{array}$ & $\begin{array}{l}-, 604 * * \\
, 000 \\
131\end{array}$ & $\begin{array}{l}-, 589 * * \\
, 000 \\
131\end{array}$ \\
\hline LBM & $\begin{array}{l}, 030 \\
, 730 \\
131\end{array}$ & $\begin{array}{l}-, 031 \\
, 726 \\
131\end{array}$ & $\begin{array}{l}-, 012 \\
, 894 \\
131\end{array}$ & $\begin{array}{l}, 169 \\
, 054 \\
131\end{array}$ & $\begin{array}{l}, 005 \\
, 952 \\
131\end{array}$ & $\begin{array}{l}1 \\
131\end{array}$ & $\begin{array}{l}, 013 \\
, 884 \\
131\end{array}$ & $\begin{array}{l}, 026 \\
, 772 \\
131\end{array}$ \\
\hline CMJ & $\begin{array}{l}, 367 * * \\
, 000 \\
131\end{array}$ & $\begin{array}{l}, 575 * * \\
, 000 \\
131\end{array}$ & $\begin{array}{l}, 548 * * \\
, 000 \\
131\end{array}$ & $\begin{array}{l}-, 257 * * \\
, 003 \\
131\end{array}$ & $\begin{array}{l}-, 604 * * \\
, 000 \\
131\end{array}$ & $\begin{array}{l}, 013 \\
, 884 \\
131\end{array}$ & $\begin{array}{l}, 953 * * \\
, 000 \\
131\end{array}$ & $\begin{array}{l}1 \\
131\end{array}$ \\
\hline $\mathrm{ABK}$ & $\begin{array}{l}, 383 * * \\
, 000 \\
131\end{array}$ & $\begin{array}{l}, 594 * * \\
, 000 \\
131\end{array}$ & $\begin{array}{l}, 564 * * \\
, 000 \\
131\end{array}$ & $\begin{array}{l}-, 250 * * \\
, 004 \\
131\end{array}$ & $\begin{array}{l}-, 589 * * \\
, 000 \\
131\end{array}$ & $\begin{array}{l}, 026 \\
, 772 \\
131\end{array}$ & $\begin{array}{l}1 \\
131\end{array}$ & $\begin{array}{l}, 953 * * \\
, 000 \\
131\end{array}$ \\
\hline
\end{tabular}

(Correlación de Pearson, sig (bilateral), $\mathrm{N}$ )

**. La correlación es significativa al nivel 0,01 (bilateral).

*. La correlación es significante al nivel 0,05 (bilateral).

Al observar los resultados obtenidos en el análisis que compara las variables antropométricas y de condición física por categorías de edad, lo primero que se observa es un incremento progresivo en las variables antropométricas de masa corporal, talla y envergadura, a medida que se incrementa la edad. Esto resulta lógico si se tiene en cuenta que los sujetos evaluados se encuentran inmersos en pleno proceso de crecimiento, lo que conlleva esa progresiva evolución. Algo diferente ocurre en el caso del porcentaje de masa grasa, donde no aparecen diferencias entre categorías de edad, algo que, según Williams (2002), se puede deber a que en estas etapas de crecimiento el principal desarrollo se produce en el tejido 
muscular y óseo, más que en otro tipo de tejidos orgánicos, como la grasa, especialmente, en el caso de sujetos activos, como los de la presente muestra. Al comparar los resultados obtenidos en variables antropométricas con los resultados de otros autores, lo primero que se advierte es la escasez de estudios de este tipo en jugadores de categoría infantil y prejuvenil, por lo que, realizar comparaciones en este sentido es complicado; esta problemática es compartida por otros autores (Silva et al 2003), más aún, cuando se aprecia, que hecha la revisión de la literatura sólo encontramos el estudio de Massa et al. (2003), quienes evaluaron cuatro categorías, de 14 a 18 años, arrojando un valor medio, considerablemente mayor en talla, en masa corporal y en envergadura, que los del presente estudio. En el caso exclusivo de los juveniles, existen autores, como Bellendier (2001), Drinkwater et al. (2007) y Gabbett et al. (2007), este último con una muestra de colegiales, quienes analizan estas mismas variables en jugadores argentinos y australianos, obteniendo registros superiores a los de la muestra de este estudio, tanto en masa corporal como en talla y en porcentaje de grasa. El contraste en la variable de talla, también se confirma en los estudios de Cabral et al. (2008; 2013), en donde se indica una media de 1,96 m, para jugadores jóvenes brasileños de elite, valor similar al de la categoría adulta; mientras la media nuestra fue de $1,75 \mathrm{~m}$, en los voleibolistas bogotanos. Sería interesante analizar el comportamiento de estas variables en la población general de estos tres países, para ver si existen diferencias con carácter general o si éstas se reducen única y exclusivamente al ámbito de los niños practicantes de voleibol. En lo que hace referencia a las pruebas de campo que evalúan la fuerza explosiva del tren inferior y del tren superior, también se observa un incremento de los registros a medida que se progresa en categoría, obteniéndose los mejores resultados, en las tres pruebas en la categoría juvenil. Al igual que ocurría en el caso de las variables antropométricas, se atribuiría la causa de esta evolución progresiva en los cambios al nivel morfológico que se producen con la edad y a los que se suman los cambios hormonales, factores que, como ya se señaló con anterioridad, influyen directamente en el desarrollo de la fuerza (López \& Fernández, 2006), capacidad de la que dependen dichas pruebas de campo. El voleibol moderno, tanto femenino como masculino, se caracteriza por la gran altura del juego en la red y la velocidad del balón en el saque saltando y en el remate, por lo que la velocidad y la potencia para efectuar saltos es una tarea motora, dominante en este deporte (Dopsaj et al. 2012).

En la literatura revisada, se encuentra gran cantidad de estudios en voleibol femenino en jóvenes, lo que demuestra la mayor práctica de este deporte por parte de las mujeres, tendencia que es similar en Colombia. Debido, probablemente, a esta escasa práctica por parte de los varones, sólo se localizaron algunos estudios en muestras masculinas en adultos, en pruebas, como el salto vertical, pero no ocurre lo mismo en el caso de los jóvenes, puesto que no se registran investigaciones con las que esta muestra se pueda comparar. En cuanto a las posiciones de juego, se encontraron diferencias significativas en las variables antropométricas de masa corporal, talla, envergadura y porcentaje de masa grasa, siendo los resultados más elevados los del jugador R3 o central. Este jugador, por sus funciones técnico-tácticas, es especializado y requiere de gran talla y envergadura, para poder cumplir con un importante número de bloqueos, en comparación con los de sus compañeros de equipo, al tener que realizar bloqueos, tanto individuales como colectivos, en todas las posiciones de ataque, durante el partido. Igualmente, el R3 o central es un jugador que cuando va al ataque requiere de una capacidad de remate en balones rápidos, lo que implica ciertas ventajas, si su talla y envergadura son elevadas. Nuestros resultados obtenidos en masa corporal y talla coinciden con los estudios de Ciccarone et al. (2005) y Fonseca et al. (2010) realizados con juveniles italianos y brasileños, respectivamente; sin embargo, en relación al jugador C2 (colocador), estos estudios lo identificaron como el de menor masa corporal y talla, diferenciándose este dato de lo obtenido en esta investigación, en donde dicho jugador ocupa el segundo lugar en masa corporal y talla, después del jugador R3.

Otro artículo con el cual coincide lo encontrado en este estudio, en cuanto al jugador de mayor talla y masa corporal es el realizado por Marques et al. (2009), con un grupo de jugadores adultos de élite de Portugal. Con respecto a la variable de porcentaje de grasa, el jugador que reporta el valor más alto es también el R3, en el presente estudio, estando en la misma línea que las investigaciones realizadas por Salem \& Zary (2004) y Fonseca et al. (2010), en juveniles, en Brasil. En relación con las variables de talla y masa corporal por posiciones de juego, nuestros resultados difieren de los encontrados por Duncan et al. (2006), con juveniles ingleses, en donde los de mayor talla son los R4, les siguen los colocadores y, por último, los centrales. Con respecto a la masa corporal, los de mayor valor fueron los R4, seguidos por los centrales y los colocadores. Finalmente, Trajkovic et al. (2011) encontraron en un estudio con pre juveniles (15 años) serbios de élite, que los mayores valores en talla los presentan los jugadores opuestos, seguidos por los centrales o R3, los R4 y, finalmente, los colocadores. En el mismo estudio, se aprecia un orden similar en lo que corresponde a la masa corporal. A pesar de que no hubo diferencias significativas en los resultados de las pruebas de campo, cabe anotar que el jugador $\mathrm{R} 4$ fue el más rápido en la prueba t-test y que el jugador C2 (colocador), obtuvo los mejores resultados en LBM y saltos CMJ y ABK. Esto puede tener su explicación en el hecho de que en Colombia, en estos niveles de competencia, los C2 son los jugadores que corresponden a la posición de juego menos frecuente en un equipo de voleibol $y$, en general, su talla no es muy elevada, por esta razón deben 
compensar esta carencia, con trabajo de salto. Por otro lado, teniendo como referencia cada posición habitual de juego y el nivel competitivo, únicamente se encontraron diferencias significativas en la posición R4 en el salto ABK, siendo mejor el resultado de GV2.

En lo que hace referencia a las correlaciones, sin diferenciar por niveles de competencia, se observa que en los jugadores de voleibol existen correlaciones positivas y estadísticamente significativas entre la masa corporal y el resto de variables antropométricas y entre la talla y la envergadura, algo que resulta lógico, especialmente, si tenemos en cuenta el periodo de crecimiento en el que están sumidos los componentes de la muestra. En este período, se producen cambios en las proporciones corporales, en la composición corporal y en la complejidad funcional para la consecución de una plenitud física. De hecho, una de las leyes que rigen este periodo del ciclo vital es lo que se conoce como la ley de progresión y amortiguación, según la cual, durante esta etapa, se presenta un aumento de la talla acompañado de un incremento proporcional de la masa corporal. A ese incremento en la talla, se le une un incremento directamente proporcional en la envergadura, debido al crecimiento simétrico de las extremidades (Ruiz, 2004). En cuanto a las pruebas de campo, en primer lugar, se observa una correlación negativa y significativa entre la prueba de agilidad y el porcentaje de masa grasa, lo que significa que, aquellos sujetos con un mayor acumulo de grasa, obtienen peores resultados en pruebas relacionadas con la agilidad. En el voleibol existen pocas investigaciones que hayan estudiado la correlación entre ambas variables. En este sentido, Sheppard \& Young (2006) señalan que, teóricamente, factores como la grasa corporal pueden influir en el desempeño de la agilidad. Basado en los resultados obtenidos, se puede concluir que, en relación al perfil antropométrico de los jugadores jóvenes de voleibol colombianos, se observa una influencia notable de la edad cronológica en la evolución de las variables evaluadas. Dicha influencia, se puede considerar superior a la ejercida por el nivel competitivo al que pertenecen los deportistas, lo que induce a pensar que, o bien el perfil antropométrico no tiene influencia en el proceso de selección de jugadores o las diferencias de esfuerzo entre niveles aún no son suficientes para generar diferencias significativas en dichas variables. Respecto al porcentaje de masa grasa, ni el nivel competitivo ni la edad cronológica parecen tener influencia en la evolución de esta variable en jugadores de voleibol, probablemente, porque las modificaciones relacionadas con el desarrollo evolutivo están vinculadas, fundamentalmente, con otros tejidos orgánicos, como el muscular o el óseo. En lo que hace referencia al perfil de condición física de los jugadores jóvenes de voleibol, de nuevo se observa una mayor influencia de la edad cronológica en la evolución de las capacidades físicas evaluadas que del nivel competitivo. Esta influencia es especialmente evidente en el caso de los juga- dores de menor nivel competitivo, donde quizás, el entorno socio-económico tenga una influencia notable en el hecho de que los mejores resultados en algunas de las variables evaluadas se obtengan por este grupo de deportistas. Teniendo en cuenta las posiciones habituales de juego únicamente se observa un perfil antropométrico específico en el caso de los centrales, algo que puede estar directamente relacionado con las exigencias técnico-tácticas del juego, tanto las de carácter ofensivo como las de carácter defensivo. Se observa un perfil específico de condición física, en el caso de los colocadores, posiblemente, también asociado a sus funciones técnico-tácticas, siendo este el jugador que debe siempre intervenir en todas las jugadas. Existe una influencia de las variables antropométricas en el desempeño físico de los individuos que es, en especial, notable en el caso del porcentaje de masa grasa, esta influye de manera negativa en el rendimiento en las capacidades físicas analizadas.

Conflictos de intereses: El manuscrito fue preparado y revisado con la participación de todos los autores, quienes declaramos que no existe ningún conflicto de intereses que ponga en riesgo la validez de los resultados presentados. Financiación: Este estudio fue financiado por recursos propios y con la colaboración del Laboratorio de Fisiología de la Universidad Pedagógica Nacional.

\section{BIBLIOGRAFÍA}

1. BELLENDIER, J. 2001. El biotipo en el voleibol masculino. Rev. Digital Educación Física y Deportes, (Buenos Aires, Argentina) 7:40. Disponible desde Internet en: en:www.efdeportes.com/efd40/biotipo.htm (con acceso 05/09/2011).

2. BOSCO, C. 1994. La valoración de la fuerza con el test de Bosco. Deporte y entrenamiento. Ed. Paidotribo. (España). p.39-41.

3. CABRAL, B.G.; CABRAL, S.; BATISTA, G.R.; FERNANDES, J.; KNACKFUSS, M.I. 2008. Somatotipia e antropometria na seleção brasileira de voleibol. Rev. Desporte o Saúde (Brasil). 4(1):67-72.

4. CABRAL, B.G.; CABRAL, S.; VITAL, R.; COSTA DE LIMA, K.; ALCANTARA, T.; MACHADO, V.; MOREIRO, P. 2013. Prediction equation of bone age in sports initiation through anthropometric variables. Rev. Bras. Med. Esporte (Brasil). 19(2):99-103.

5. CICCARONE, G.; FONTANI, G.; ALBERT, A.; ZHANG, L.; CLOES, M. 2005. Analisi delle caratteristiche antropometriche e delle capacitá di salto di giovani pallavolisti di alto livelo. Medicina Dello Sport. (Italia). 58(1):1-15. 
6. DOPSAJ, M.; ĆOPIĆ, N.; NEŠIĆ, G.; SIKIMIĆ, M. 2012. Jumping performance in elite female volleyball players relative to playing positions: a practical multidimensional assessment model. Serbian J. Sports Sci. 6(2):61-69

7. DRINKWATER, E.J.; LAWTON, T.W.; MCKENNA, M.J.; LINDSELL, R.P.; HUNT, P.H.; PYNE, D.B. 2007. Increased number of forced repetitions does not enhance strength development with resistance training. J. Strength Cond. Res. (USA). 21(3):841-847.

8. DUNCAN, M.; WOODFIELD, L.; AL-NAKEEB, Y. 2006. Anthropometric and physiological characteristics of junior elite volleyball players. Br J. Sports Med. (Inglaterra). 40:649-651.

9. DURKOVIC, T.; MARELIC, N.; REŠETAR, T. 2012. Morphological differences of premier league volleyball players according to their playing roles. Hrvat. Športskomed. Vjesn. (Croacia). 27:72-78.

10. FAULKNER, J.A. 1968. Physiology of swimming and diving. En: Falls H. Ed. Baltimore Academic Press (Baltimore). p. 415-446.

11. FONSECA, T.; ROQUETTI, P.; FERNÁNDEZ, J. 2010. Análisis del perfil antropométrico de jugadores de la selección brasileña de voleibol infanto juvenil. Int. J. Morphol. (Brasil) 28(4):1035-1041.

12. GABBETT, T.; GEORGIEFF, B.; DOMROW, N. 2007. The use of physiological, anthropometric, and skill data to predict selection in a talent-identified junior volleyball squad. J. Sports Sci. (Australia) 25(12):13371344.

13. GABBETT, T. 2008. Do skill-based conditioning games offer a specific training stimulus for junior elite volleyball players? J. Strength Cond. Res. (USA). 22(2):509-517.

14. GAURAV, V.; SINGH, M.; SINGH, S. 2011. A comparative study of somatic traits and body composition between volleyball players and controls. Indian J. Sci. Techn. 4(2):116-118.

15. LÓPEZ, J.; FERNÁNDEZ, A. 2006. Fisiología de ejercicio. 3a Edición. Ed. Médica Panamericana. (España). 1005p.

16. MARQUES, M.C.; VAN DEN TILLAR, R.; GABBETT, T.J.; REIS, V.M.; GONZÁLEZ-BADILLO, J.J. 2009. Physical fitness qualities of profesional volleyball players: determination of positional differences. J. Strength Cond. Res. 23(4):1106-1111.

17. MARTíNEZ, E.J. 2012. Pruebas de aptitud física. $2^{\mathrm{a}}$ Ed. Ed. Paidotribo. (España). p.143.

18. MASSA, M.; SILVEIRA, M.T.; RIGOLIN, L.R.; UEZU, R. 2003. Análise de referenciais cineantropométricos de atletas de voleibol masculino envueltos em procesos de promocao de talentos. Rev. Mackenzie Edu. Fís. Esporte. (Brasil). 2(2):101-113.

19: MAZZA, J.C. 2010. Antropométrica. Biosystems Books Servicio Educativo. (Argentina). 273p.

20. PAUOLE, K.; MADOLE, K.; GARHAMMER, J.; LACOURSE, M.; ROZENEK, R. 2000. Reliability and Validity of the T-Test as a Measure of Agility, Leg Power, and Leg Speed in College-Aged Men and Women. J. Strength Cond. Res. 14(4):443-450.

21. PEÑA, J.; RODRÍGUEZ, J.; BUSCÁ, B.; SERRA, N. 2013. Which skills and factors better predict winning and losing in high-level men's volleyball? J. Strength Cond. Res. 27(9):2487-2493.

22. RUIZ, L.M. 2004. Desarrollo motor y actividades físicas. Ed. Gymnos (España). p.77-114.

23. SALEM, M.; ZARY, J.C.F. 2004. Evolução do perfil somatotípico da seleção brasileira de voleibol masculino juvenil de 2000/2003. Rev. Educ. Fís. (Brasil). 128(1):41-51.

24. SHEPPARD, J.; YOUNG, 2006. Agility literature review: Classifications, training and testing. J. Sport Sci. (Australia). 24(9):919-932.

25. SILVA, L.R.R.; BOHME, L.T.; UEZU, R.; MASSA, M. 2003. A utilização de variáveis cineantropométricas no proceso de detecção, seleção e promoçãode talentos no voleibol. Rev. Bras. Ciênc. Mov. 11(1):69-76.

26. SMITH, D.J.; ROBERTS, D.; WATSON, B. 1992. Physical, physiological and performance differences between Canadian national team and universiade volleyball players. J. Sports Sci. 10:131-138.

27. TOLEDO, F.C, CLAUDIO, L; SILVA, D.P; ROQUETTI, F.P; FERNANDES, F.J. 2000. Perfil dermatoglífico, somatotípico y de la fuerza explosiva de atletas de la selección brasileña de voleibol. Fitness Perfom. J. (Brasil). 7(1):35-40. 
28. TORRES, J.; UREÑA, A.; GONZÁLEZ, M.; MORENTE, C.J. 1993. Manual del preparador de voleibol. Nivel II. Federación Andaluza de Voleibol. (España). 774p.

29. TRAJKOVIC, N.; MILANOVIC, Z; SPORIŠ, G; RADISAVLJEVIC, M. 2011. Positional differences in body composition and jumping performance among youth elite volleyball players. Acta Kinesiol. (Serbia). 5(1):62-66
30. WILLIAMS, M. 2002. Nutrición para la salud, la condición física y el deporte. Edit. Paidotribo (España). p.323.

Recibido: Noviembre 5 de 2013

Aceptado: Febrero 13 de 2014

\section{Como Citar:}

González, Y.; Sedano, S.; Fernández, J.; Díaz, H. 2014. Estudio comparativo de factores antropométricos y de condición física en jugadores jóvenes de voleibol colombiano. Rev. U.D.C.A Act. \& Div. Cient. 17(1): 53-63. 
